\title{
Epigallocathechin gallate, polyphenol present in green tea, inhibits stem-like characteristics and epithelial-mesenchymal transition in nasopharyngeal cancer cell lines
}

Chien-Hung Lin ${ }^{1,2}$, Yao-An Shen ${ }^{3}$, Peir-Haur Hung ${ }^{4}$, Yuan-Bin Yu ${ }^{5}$ and Yann-Jang Chen ${ }^{1,6,7^{*}}$

\begin{abstract}
Background: Previous studies have demonstrated that the consumption of green tea inhibits the growth of various cancers. Most cancers are believed to be initiated from and maintained by a small population of cancer stem-like cells (CSC) or tumor-initiating cells (TIC) that are responsible for tumor relapse and chemotherapeutic resistance. Although epigallocathechin gallate (EGCG), the most abundant catechin in green tea, has been reported to induce growth inhibition and apoptosis in some cancer cells, its effect on CSC is undefined. In this study, we enriched CSC by the sphere formation, and provided an efficient model for further experiments. Using this method, we examined the effects of EGCG regulating the nasopharyngeal carcinoma (NPC) CSC and attempted to elucidate the possible mechanisms.
\end{abstract}

Methods: NPC TW01 and TW06 cell lines were enriched by sphere formation and characterized their phenotypical properties, such as invasion capacity, epithelial-mesenchymal transition (EMT) and gene expression were analyzed by quantitative real-time reverse transcription polymerase chain reaction (q-RT-PCR). EGCG-induced growth inhibition in the parental and sphere-derived cells was determined by MTT and bromodeoxyuridine (BrdU) assay. EGCG-induced apoptosis was analyzed by flow cytometry with Annexin $V$ and PI staining. The effects of EGCG on sphere-derived cell tumorigenicity, migration and invasion were determined by soft agar assay, wound healing, and cell invasion assay. The alternation of protein expression regulated by EGCG on these sphere-derived cells was assessed by immunofluorescence staining and western blot.

Results: NPC sphere-derived cells grown in serum-free non-adherent culture showed increased expression of stem cell markers and EMT markers compared to parental cells grown in conventional culture. Although EGCG induced growth inhibition and apoptosis in the parental cells in a dose-dependent manner, it was not as effective against spheres. However, EGCG potently inhibited sphere formation and can eliminate the stem cell characteristics of NPC and inhibit the epithelial-mesenchymal transition (EMT) signatures.

Conclusions: Overall, these findings show that NPC cells with sphere formations possess the properties of CSC. Using this model, we found that EGCG regulated NPC CSC, their self-renewal capacity, and inhibited their invasive characteristics. It supports the pivotal role of EGCG as a dietary compound targeting NPC and may decrease recurrence and metastasis in nasopharyngeal carcinoma cells.

Keywords: Epigallocathechin gallate, Nasopharyngeal carcinoma, Cancer stem cells, Stem cells, Epithelial-mesenchymal transition

\footnotetext{
* Correspondence: yjchen@ym.edu.tw

${ }^{1}$ Institute of Clinical Medicine, National Yang-Ming University, Taipei, Taiwan

${ }^{6}$ Department of Pediatrics, Renai Branch, Taipei City Hospital, Taipei, Taiwan

Full list of author information is available at the end of the article
} 


\section{Background}

Nasopharyngeal carcinoma (NPC) arises from the nasopharyngeal epithelial cells with high prevalent rates in Southeast China and Taiwan. According to the World Health Organization (WHO) histological classification, NPC is categorized into three groups: keratinizing squamous cell carcinoma (SCC, type I), nonkeratinizing carcinoma (NKC, type II) and undifferentiated carcinoma (UC, type III) [1]. NPC has high metastatic potential with frequent initial dissemination to regional lymph nodes, and distant metastases at diagnosis, and this underlies the majority of NPC-related death $[2,3]$. Advanced-stage NPC might have chemotherapy resistance and radio-resistance. The prognosis remains poor in a significant number of NPC patients with relapse or metastatic diseases.

Evidences support the hypothesis that tumors contain small subpopulations of cells called cancer stem-like cells (CSC) or tumor-initiating cells (TIC) $[4,5]$. These cells are generally thought of as self-renewing cells that are able to reinitiate a tumor for several generations and can give rise to a spectrum of differentiated cells. CSCs are able to proliferate and self-renew extensively because of their ability to express anti-apoptotic and drug-resistant proteins, thus sustaining tumor growth [6]. Presumed cancer stem-like cells had been proposed in nasopharyngeal tumors by side population and surface markers $[7,8]$.

To eradicate NPC and prevent recurrence, it is imperative that NPC CSC should be specifically and efficiently inhibited. In addition, CSC undergoing metastasis often expresses epithelial-to-mesenchymal transition (EMT) and the high EMT markers have been observed in dissemination of carcinoma cells from primary epithelial tumors [9]. The interaction between stemness characteristics and EMT expression has advanced results in recent studies. Researches have shown that EMT acquires CSC properties, increases cancer cell tumorigenicity and shows a crucial link to metastasis [10-12].

One assay for enriched and characterized stem cells is based on the sphere-generated cells growing in serum free suspension. These cells may represent a tumorinitiating subpopulation with an ability of self-renewal, and proliferate unlimitedly [13]. We demonstrated that the sphere-derived cells of NPC are more invasive, chemoresistant, and express anti-apoptosis and EMT genes compared to parental monolayer cells and these served as a model for designing experiments.

The cancer preventive effects of green tea are widely supported by results from epidemiological, cell culture, animal and clinical studies. This property has mainly been attributed to the most prevalent tea polyphenol, epigallocathechin gallate (EGCG), which has potent capacity in inhibiting cancer growth and inducing apoptosis in various cancers by several mechanisms. It causes cell cycle deregulation and apoptosis of cancer cells through NF-kappa B inhibition and activation of caspases [14,15]. It inhibited expressions of $\mathrm{Bcl}-2$ and $\mathrm{Bcl}-\mathrm{XL}$ and induced expressions of Bax, Bak and Bcl-XS [16,17]. In addition, EGCG inhibited angiogenesis and metastasis through repressing the expression of vascular endothelial growth factor (VEGF), and matrix metalloproteinases (MMP)-2 and MMP-9 in prostate cancer [18]. It is also an important preventive agent for declining cancer incidence, and has synergistic effects on cancer cell responses to conventional chemotherapy [19-21].

Recent studies have shown that some dietary compounds have potential to act against CSC through several regulatory mechanisms [22-24]. However, EGCG involved in possible mediated molecular mechanisms and therapeutic targets in regulating CSC has been largely unexplored.

This study investigated if EGCG can target NPC CSC by using sphere-derived cells, and realized the underlying molecular mechanisms.

\section{Methods \\ Cell culture \\ Parental monolayer cell culture}

NPC TW01 (WHO type I, keratinizing squamous cell carcinoma) and TW06 (WHO type III, undifferentiated carcinoma) cell lines established from Taiwanese were cultured in $10 \mathrm{~cm}^{2}$ dishes with Dulbecco's Modified Eagle Medium (DMEM) (Invitrogen Carlsbad, CA) and 10\% FBS (BIOIND, Kibbutz Beit Haemek, Israel), 1\% sodium pyruvate (BIOIND), 1\% penicillin, streptomycin, amphotericin (PSA, BIOIND) and 1\% Non-Essential Amino Acids (NEAA, BIOIND). The cells were incubated at $37^{\circ} \mathrm{C}$ in a humidified atmosphere of $5 \% \mathrm{CO} 2$.

\section{Non-adherent culture}

TW01 and TW06 parental cells were seeded nonadhesively in 6-well culture dishes coated with thin agarose at a density of $2 \times 10^{4} / \mathrm{mm}^{3}$ in serum-free DMEM/ F12 medium (Invitrogen). Each treatment was carried out in triplicates. The culture medium was changed every other day until sphere formation. After 7 to 10 days, the spheres were collected by filtration through a $70 \mu \mathrm{m}$ mesh for the following experiments.

\section{RNA extraction and quantitative real-time $R T-P C R$}

The total RNA was isolated with Trizol reagent (Invitrogen). First-strand cDNA was reverse transcribed (RT) according to the manufacturer protocols. Relative levels of mRNA were determined by qPCR using a real time PCR system. Several stemness genes and epithelialmesenchymal transition markers were analyzed. The primer sequences used for quantitative real-time RT-PCR are shown in Table 1. GAPDH was the endogenous 


\begin{tabular}{ll}
$\begin{array}{l}\text { Table } 1 \text { The primer sequences for q-RT PCR used in this } \\
\text { study }\end{array}$ \\
\hline Gene & Sequence \\
\hline GAPDH & Forward : 5'-ACGGGAAGCTCACTGGCATGG-3' \\
& Reverse : 5'-GGTCCACCACCCTGTTGCTGTA-3' \\
Sox-2 & Forward : 5'-CGAGTGGAACTTTGTCGGA-3' \\
& Reverse: 5'-TGTGCAGCGCTCGCAG-3' \\
Oct-4 & Forward: 5'-GTGGAGAGCAACTCCGATG-3' \\
& Reverse: 5'-TGCTCCAGCTTCTCCTTCTC-3' \\
C-Myc & Forward: 5'-GGAACGAGCTAAAACGGAGCT-3' \\
& Reverse: 5'-GGCCTTTCATTGTTTCCAACT-3' \\
KIf-4 & Forward: 5'-CCGCTCCATTACCAAGAGCT-3' \\
& Reverse: 5'-ATCGTCTTCCCCTCTTTGGC-3' \\
E-Cad & Forward: 5'-TGCCCAGAAAATGAAAAGG-3' \\
& Reverse: 5'-GTGTATGTGGCAATGCGTC-3' \\
Vim & Forward: 5'-GAGAACTTGCCGTTGAAGC-3' \\
& Reverse: 5'-GCTTCCTGTAGGTGGCAATC-3' \\
Snail & Forward: 5'-CTCCAGCAGCCCTACGAC-3' \\
& Reverse: 5'-CGGTGGGTTGAGGATCT-3' \\
N-Cad & Forward: 5'-AGGGTGGACGTCATTGTAGC-3' \\
& Reverse: 5'-CTGTGGGGTCTGTCAGGAT-3' \\
& Forward: 5'-CGAGTCCGCAGTCTTACGAG-3' \\
& Reverse: 5'-TCTGGAGGACCTGGTAGAGG-3' \\
\hline
\end{tabular}

reference. cDNA was subjected to PCR for 35 cycles of $94^{\circ} \mathrm{C}$ for $30 \mathrm{~s}, 55^{\circ} \mathrm{C}$ for $30 \mathrm{~s}$, and $72^{\circ} \mathrm{C}$ for $45 \mathrm{~s}$. q-PCR was performed using an ABI PRISM ${ }^{\circledR} 7900 \mathrm{HT}$ system (Applied Biosystems, Foster City, CA).

\section{Cell proliferation assay by MTT and BrdU assay}

The effect of EGCG on proliferation of NPC TW01, TW06 parental and sphere-derived cells was examined by MTT [3-(4, 5-dimethylthiazol-2-yl)-2, 5-diphenyltetrazolium bromide] assay. Cells $\left(2.5 \times 10^{3}\right.$ per well $)$ were seeded in 96-well plates and allowed to grow for $24 \mathrm{~h}$. Then EGCG or DMSO (vehicle control) was added after plating onto adherent cells at specified concentrations. Each treatment was triplicated. After $72 \mathrm{~h}, 20 \mu \mathrm{l}$ of MTT solution ( $5 \mathrm{mg} / \mathrm{ml}$; Sigma, St. Louis, MO) was added to each well and incubated for $4 \mathrm{~h}$ at $37^{\circ} \mathrm{C}$. The MTT formazan crystal was then dissolved in DMSO, and the absorbance was measured by a microplate reader (Bio-Rad 680, Bio-Rad Laboratories, Hercules, CA) at a wavelength of $570 \mathrm{~nm}$.

Cell proliferation was also determined by bromodeoxyuridine (BrdU) incorporation analysis (Roche Applied Science, Mannheim, Germany). Briefly, cells were plated in 96-well plates $\left(2.5 \times 10^{3}\right.$ per well) for $24 \mathrm{~h}$ and BrdU incorporated in the newly-synthesized DNA of EGCG- treated or vehicle was quantified after $72 \mathrm{~h}$. After labeling with BrdU $(10 \mu \mathrm{M})$ for $2 \mathrm{~h}$, cells were fixed, incubated with anti-BrdU-peroxidase $(10 \mu \mathrm{l} /$ well $)$ for $1 \mathrm{~h}$ and 30 $\mathrm{min}$, and followed by the addition of trimethyl benzidine substrate to measure the amount of incorporated BrdU. Absorbance of each well was measured at wavelength of $450 \mathrm{~nm}$.

\section{Apoptosis assessed by flow cytometry}

TW01 parental and sphere-derived cells were treated after plating in the monolayer with different concentrations of EGCG for $72 \mathrm{~h}$ respectively. Cells were washed twice with cold PBS and then resuspended in 1X Binding Buffer at a concentration of $1 \times 10^{6}$ cells $/ \mathrm{ml}$. Transfer of $100 \mu$ lof solution $\left(1 \times 10^{5}\right.$ cells $)$ to a $5 \mathrm{ml}$ culture tube was performed and $5 \mu \mathrm{l}$ of FITC Annexin V and $5 \mu \mathrm{l}$ propidium iodide (PI) (BD Pharmingen, San Diego, CA) were then added. The cells were gently vortexed, and incubated for $15 \mathrm{~min}$ at $\mathrm{RT}\left(25^{\circ} \mathrm{C}\right)$ in the dark. A $400 \mu \mathrm{l}$ of $1 \mathrm{X}$ Binding Buffer was added to each tube. Analysis was performed by FC500 flow cytometer (Beckman Coulter, Brea CA) to identify the subpopulations of the apoptosis cells within $1 \mathrm{hr}$.

\section{Soft agar clonogeneic assay}

The bottom of each well $(35 \mathrm{~mm})$ of 6-well culture dish was coated with $2 \mathrm{ml}$ agar mixture (DMEM, 10\% [v/v] FCS, $0.6 \%[\mathrm{w} / \mathrm{v}]$ agar). After the bottom layer solidified, $2 \mathrm{ml}$ top agar-medium mixture (DMEM, 10\% [v/v] FCS, $0.3 \%[\mathrm{w} / \mathrm{v}]$ agar) containing $2 \times 10^{4}$ sphere-derived cells with different concentrations of EGCG was added and incubated at $37^{\circ} \mathrm{C}$ for 2 weeks. At the end of the incubation period, the number of colonies was counted using microscope after staining with the crystal violet.

\section{Wound healing assay}

NPC TW01 parental and sphere-derived cells were seeded in 6-well plates and grown to confluence, and then added with EGCG. Monolayers were scraped with a micropipette and photographed at the beginning of the assay at $0 \mathrm{~h}$ and at $24 \mathrm{~h}$ in respective conditions.

\section{Cell invasion assay}

Cultrex $^{\circledR} 96$ Well Collagen IV Cell Invasion Assay (Trevigen Inc. Gaithersburg, MD) was used according to the manufacturer instructions. Briefly, cells were cultured to $80 \%$ confluence, serum starved over-night, then harvested and re-suspended to a density of $5 \times 10^{4}$ cells $/ \mathrm{ml}$ in serum-free medium. Cell suspension of $50 \mu \mathrm{l}$ was added to the top chamber with and without EGCG, and $150 \mu \mathrm{l}$ of media with FBS was added to the lower well. The top invasion chamber was coated with a membrane of $50 \mu \mathrm{l} \mathrm{BME} \mathrm{(basement} \mathrm{membrane} \mathrm{extract)} \mathrm{solution.}$ The chambers were incubated at $37^{\circ} \mathrm{C}$ in a humidified 
environment for $24 \mathrm{~h}$. The following day, cells invading the lower wells were detected by calcein-AM staining followed by fluorescence measurement on a microplate reader at $485 \mathrm{~nm}$ excitation and $520 \mathrm{~nm}$ emissions.

\section{Immunofluorescence staining}

Cells were cultured on sterile glass coverslips in 6-well plates and fixed in $4 \%$ paraformaldehyde. After washing with PBS, the cells were permeabilized using $0.1 \%$ Triton X-100 solution and blocked with 10\% BSA. The slides were incubated overnight at $4^{\circ} \mathrm{C}$ with the primary antibodies, followed by incubation with the fluorescentlylabeled secondary antibodies at room temperature for $1 \mathrm{~h}$. Slides were counterstained with 4'-6-diamidino-2- phenylindole (DAPI) and analyzed under a fluorescent microscope (Leica DM 4000B).

\section{Western blot analysis}

Western blot was performed to detect proteins involved in EMT (E-cadherin, Snail, and Vimentin). Cells were lysed in a RIPA buffer containing a 1X protease inhibitor cocktail, and protein concentrations were determined using the Bradford assay (Bio-Rad, Philadelphia, PA). Proteins were separated by $10 \%-12.5 \%$ SDS/PAGE and transferred to membranes (Millipore, Bedford, MA) at $55 \mathrm{~V}$ for $4 \mathrm{~h}$ at $4^{\circ} \mathrm{C}$. After blocking in $5 \%$ non-fat dry milk in TBS, the membranes were incubated with primary antibodies at 1:1000 dilution in TBS overnight at $4{ }^{\circ} \mathrm{C}$, washed 3 times with TBS-Tween 20 , and then
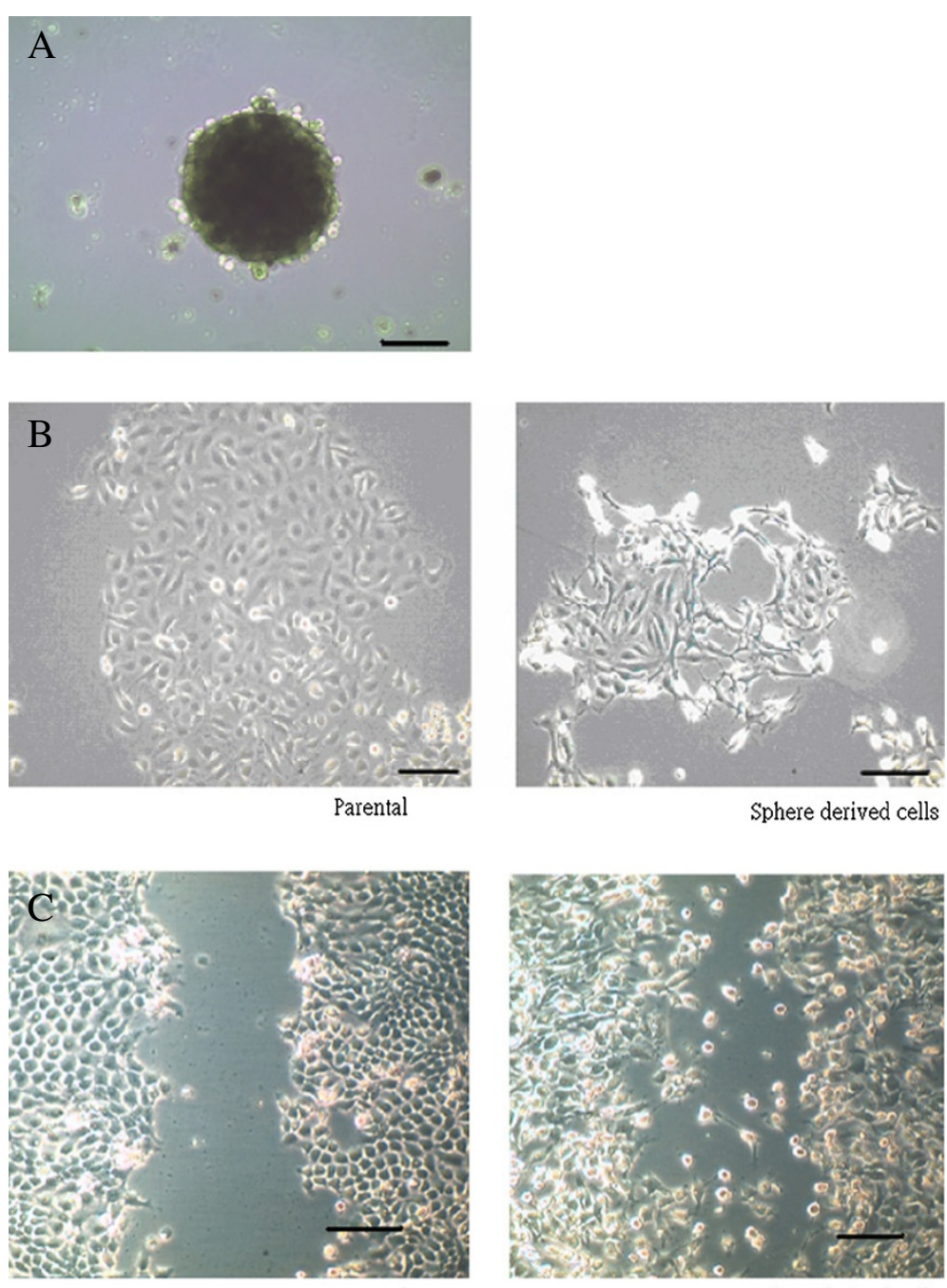

Sphere derived cells

Figure 1 NPC sphered cells showed different properties compared to parental cells. (A) NPC TW01 sphere grew in serum free non-adherent condition (B) Different cell shape of TW01 parental and sphere-derived cells were noted when cultured adherently in 10\% serum for $48 \mathrm{~h}$. Sphered cells present in mesenchymal shape. (C) Sphere-derived cells present more powerful migratory behavior after $12 \mathrm{~h}$ of scraping (Scale bars show $100 \mu \mathrm{m}$ ). 


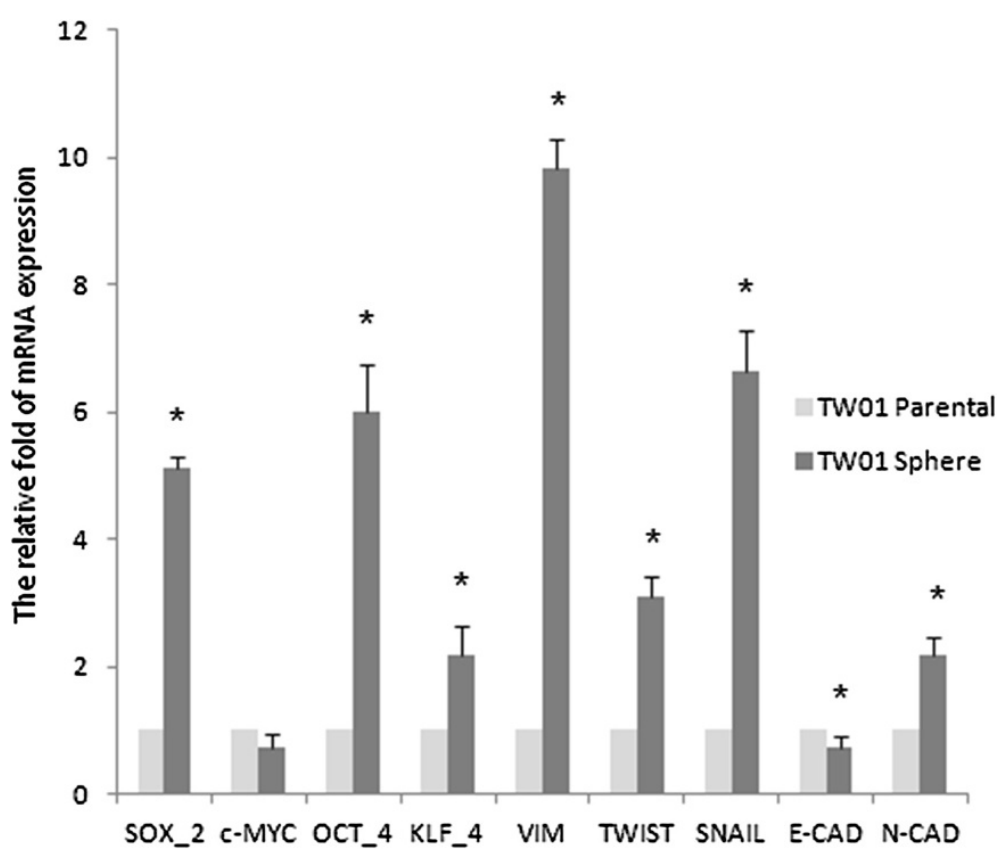

Figure 2 mRNA expressions of stemness genes and EMT markers analyzed by q-RT-PCR. The amounts of Sox-2, Oct-4, Klf-4 and EMT markers Vimetin, Twist, Snail and N-cad were increased in TW01 sphere-derived cells as compared to parental cells. The internal control is housekeeping gene-GAPDH and every gene expression of sphere-derived cells was normalized with parental cells. Data represent mean \pm SD. * means significantly different from respective controls, $p<0.05$.
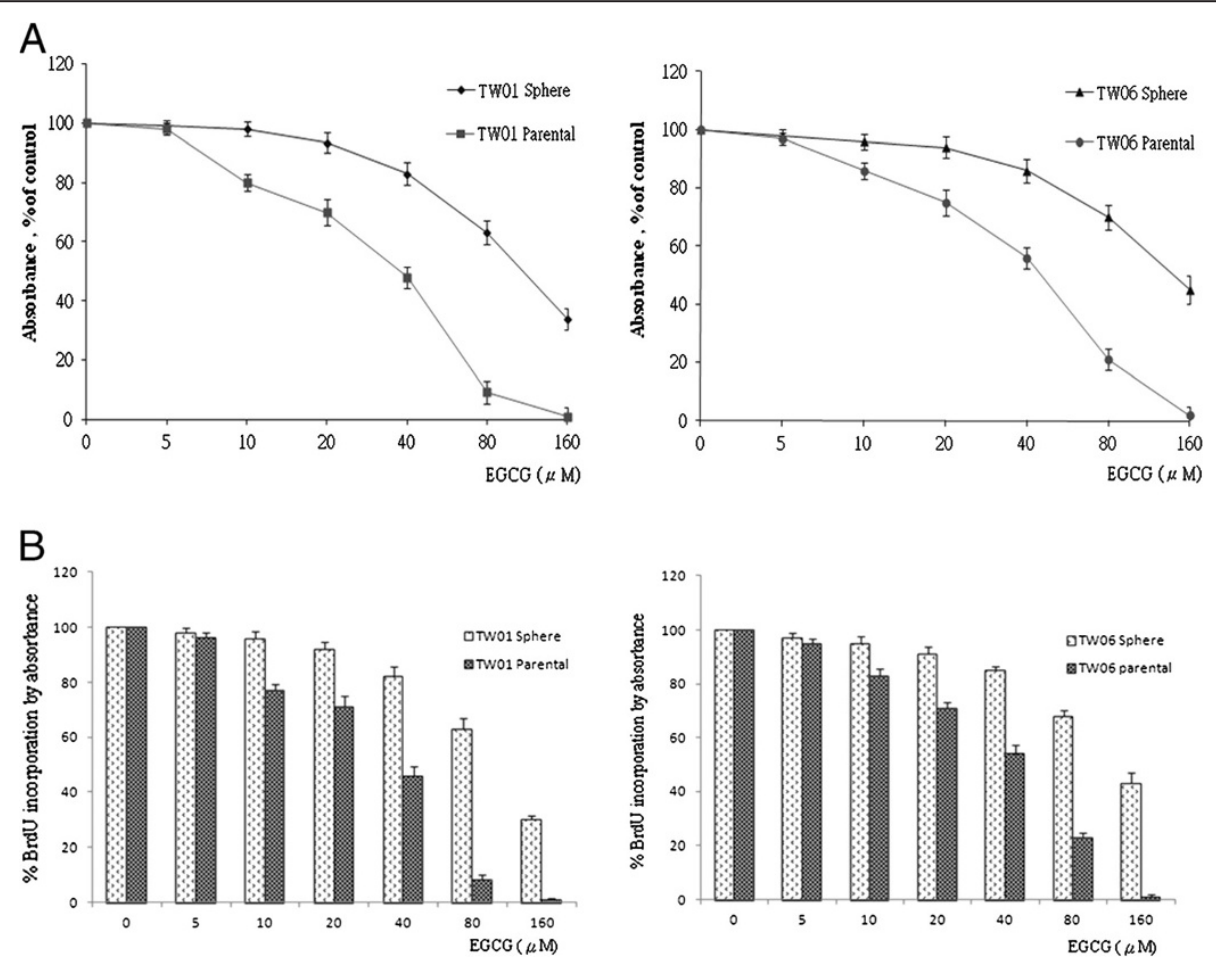

Figure 3 Effect of EGCG on NPC cell proliferation. EGCG had effects on cell proliferation and induced growth inhibition of NPC parental and sphere-derived cell in a concentration dependent manner. However, sphere-derived cells showed relative resistance to EGCG ( $\mu M)$ compared to parental cells. (A) MTT assay and (B) BrdU assay. Both assays showed similar results. 

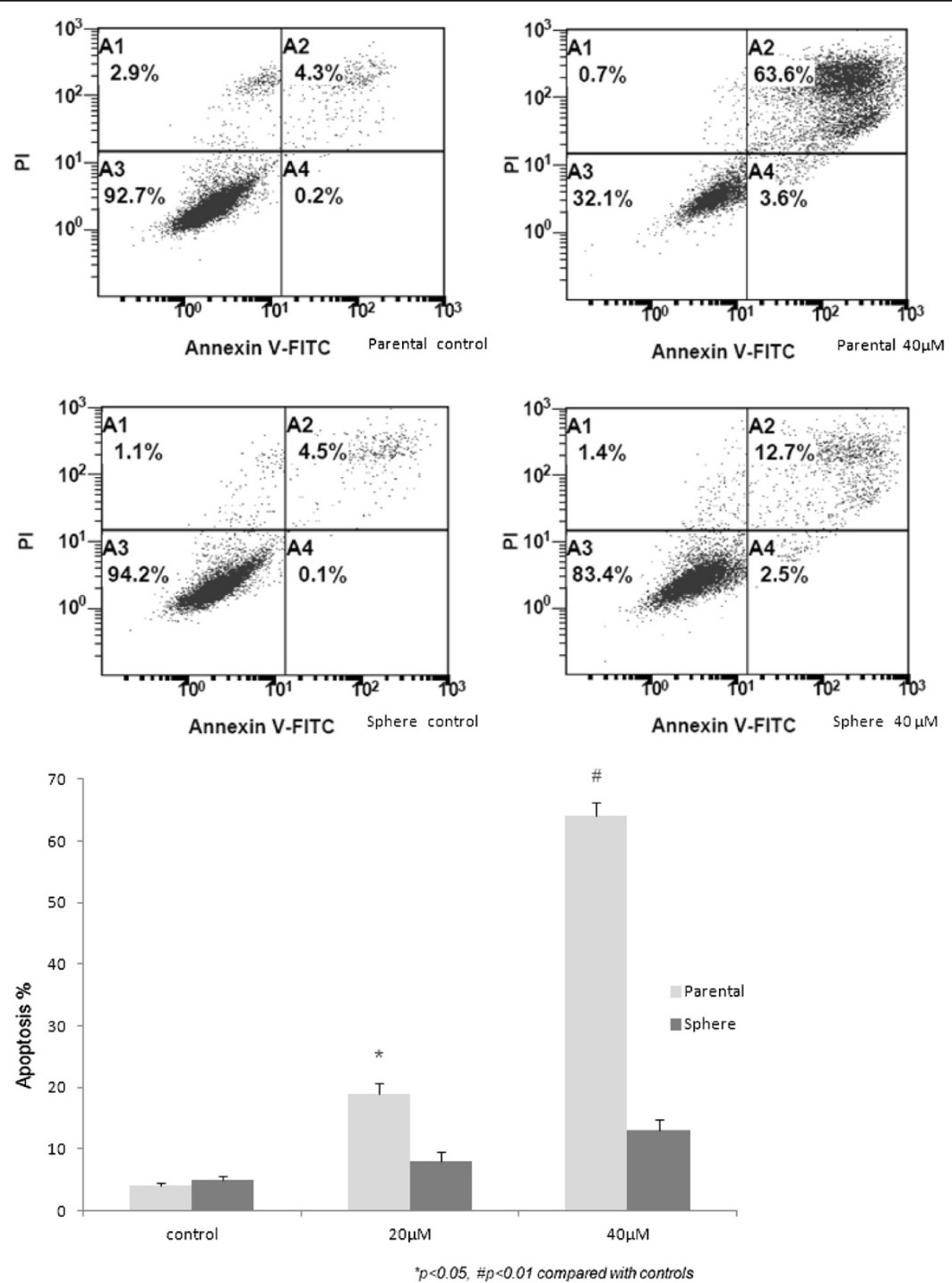

Figure 4 Apoptosis effect of EGCG on NPC cells. The upper panel shows the apoptosis frequency of TW01 parental cell increased from 4.3\% up to $63.6 \%$ after $40 \mu \mathrm{M}$ EGCG treatment, while only up to $12.7 \%$ is noted in the sphere-derived cells. The lower panel shows EGCG-induced TW01 parental cell apoptosis was significantly correlated with a dose-dependent trend but was not as effective in TW01 sphere-derived cells.

incubated with secondary antibodies conjugated with horseradish peroxidase at 1:5000 dilution in TBS for $1 \mathrm{~h}$ at room temperature. Membranes were washed again in TBS-Tween 203 times at room temperature. Protein bands were visualized on X-ray film using an enhanced chemiluminescence detection system. The primary antibodies against E-cadherin and Vimentin were purchased from Sigma-Aldrich (St Louis, MO). Snail from Abcam (Cambridge, MA) and used at a dilution of 1:1000.

\section{Statistical analysis}

Data are expressed as the mean \pm SD from a minimum of 3 separate experiments. The differences between 2 groups were analyzed by the Student $t$-test. The differences among 3 groups were analyzed by one or two way ANOVA, statistical significance was considered as $p<0.05$. Statistical analyses were performed with SPSS for Windows version 14.0.

We had ethical approval. Our experimental research performed with the approval of an appropriate ethics 
committee. Our research carried out in vitro, not on humans and not on animals.

\section{Results}

NPC Cells with ability to form spheres acquired features of CSC and underwent EMT

First, we investigated NPC cell lines containing cells with self-renewal that form tumor spheres, and have the ability to migrate, invade, and express the EMT phenotype (Figure 1). NPC cells showed increased expression of stem cell markers Sox-2, Oct-4, and Klf-4 in spherederived cells grown in serum-free non-adherent culture compared to parental cells grown in conventional culture. In addition, the expression of mRNA of the transcription factors involved in EMT, such as Twist, Snail, and mesenchymal makers, such as Vimetin and Ncadherin were also increased in sphere-derived cells compared to the parental cells. However, a decreased expression of E-cadherin in sphere-derived cells was detected (Figure 2).

\section{Effects of EGCG on TW01, TW06 growth, and apoptosis} The proliferation-inhibition effects of EGCG with different concentrations in NPC TW01 and TW06 cell lines were evaluated by MTT assay and BrdU assay. Both results showed EGCG-induced inhibition of TW01 and TW06 proliferation in a concentration dependent manner (Figure 3). However, sphere-derived cells showed more resistance to the EGCG-inhibition effect compared to parental cells, and were less effective in inducing growth inhibition.

The apoptosis effect modulated by EGCG was detected by flow cytometry with Annexin V and PI double staining. The results showed increased apoptotic activity in TW01 parental cells treated with $40 \mu \mathrm{M}$ EGCG for $72 \mathrm{~h}$ but this was not apparent in TW01 sphere-derived cells (Figure 4). As compared to the control, EGCG-induced TW01 parental cell apoptosis significantly correlated to dose-dependent trends, however, this did not occur in TW01 sphere-derived cells.

\section{Inhibition on NPC sphere-derived cell colony formation,} migration, and invasion by EGCG

TW01 and TW06 sphere-derived cells were grown in agar and various doses of EGCG were added for 2 weeks. Colonies were counted at the end of the incubation period, and we found that EGCG inhibited the growth of colonies in a dose-dependent manner (Figure 5). The results suggest that EGCG can inhibit the self-renewal and tumorigenicity capacity of NPC CSC.

Wound-healing assay was performed to assess whether EGCG affected TW01 sphere-derived cell migration. The control group without EGCG treatment produced marked cell migration in the wound area $24 \mathrm{hr}$ after wounding, but wounds treated with EGCG showed significant delays under the same conditions (Figure 6). By the cell invasion assay, the invasive ability was significantly inhibited in sphere-derived cells treated with EGCG in a dose-dependent manner (Figure 7).

\section{EGCG regulated the expression of stem cell genes in}

\section{sphere-derived cells}

Increased expression of stemness genes-Sox-2, Klf-4, and Oct- 4 was detected in sphere-derived cells compared to parental cells. We next examined the effects of EGCG on the mRNA expression levels of these factors. TW01 sphere-derived cells were treated with or without EGCG. After a $36 \mathrm{~h}$ incubation period, cells were harvested and the expression of Sox-2, Klf-4, and Oct-4 was measured by the real-time RT-PCR (Figure 8 ). The results showed EGCG inhibited the expression of Klf-4 and Oct-4 in sphere-derived cells. However, the expression of Sox-2 was not affected by EGCG.

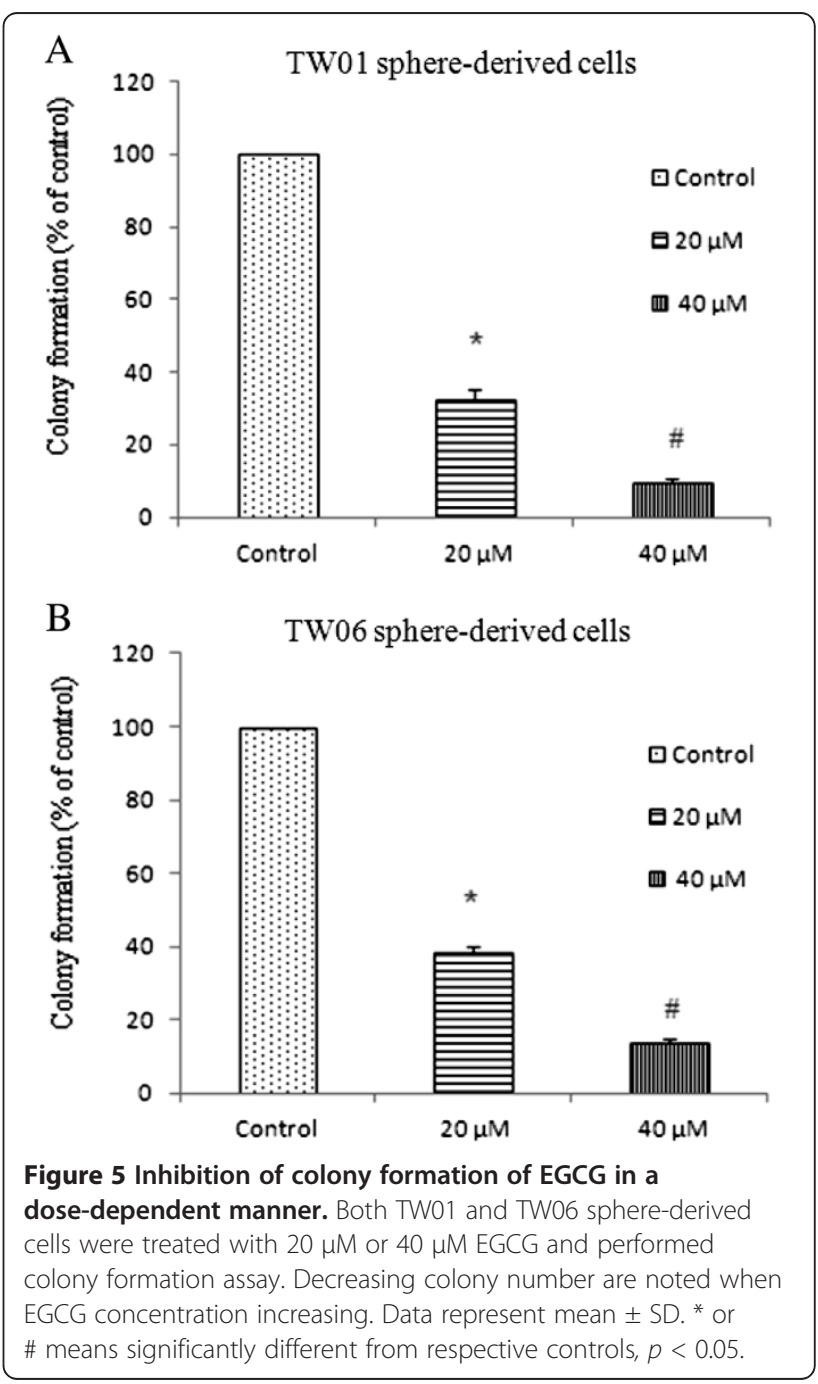



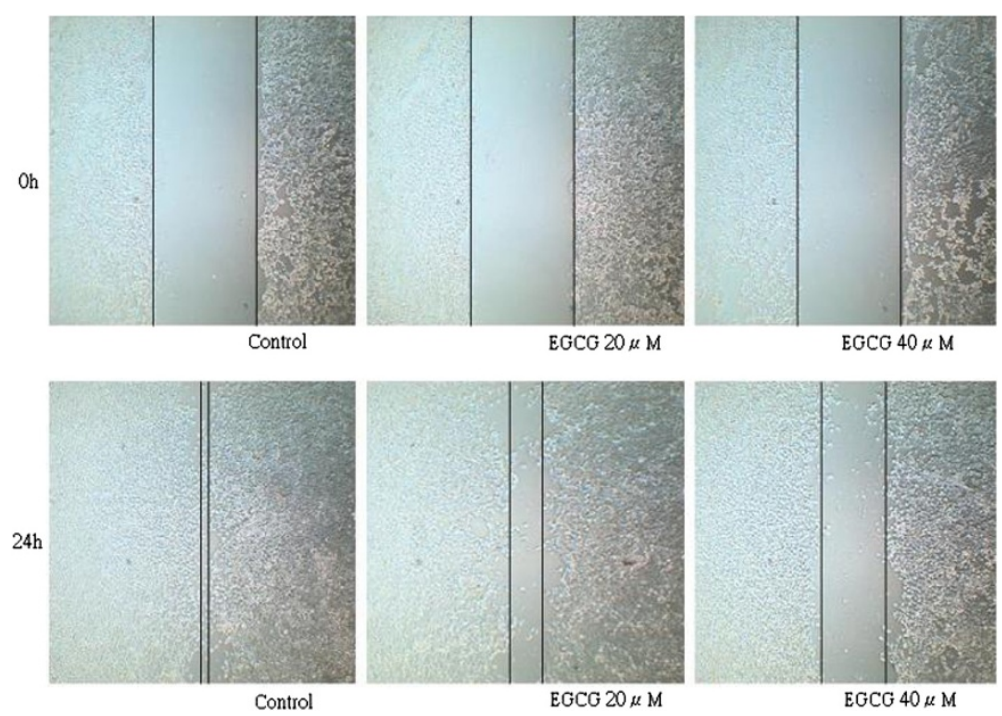

Figure 6 EGCG inhibited cell migration shown by wound healing assay. TW01 sphere-derived cells produced marked cell migration in the wound area $24 \mathrm{~h}$ after wounding, but wounds treated with EGCG showed significant delays under the same conditions.

\section{EGCG regulated the expression of EMT-related protein} Due to change of cell shape and EMT marker expression, NPC sphered cells might have undergone EMT change. We then studied whether the expression of EMT markers regulated by EGCG in TW01 spherederived cells correlated with migration and invasive property changes. Immunofluorescence staining in sphere-derived cells treated with or without EGCG was performed and showed that vimentin expression was decreased in EGCG-treated cells (Figure 9A C). Moreover, in western blotting, EGCG significantly inhibited the levels of Snail, Vimentin and increased E-Cadherin expression in a dose-dependent manner (Figure 9D). Our results showed that the invasion and migration ability of TW01 sphere-derived cells was inhibited by EGCG in relation to regulating EMT-related protein expression.

\section{Discussion}

Unlimited growth, recurrence, and metastasis of cancer have been related to the behavior of CSC that can be identified by in vitro assay. Cancer stem cells undergoing metastasis that have been demonstrated to have EMT marker expressions associated with invasive and migratory properties easily invade the surrounding tissues. This invasive type of cancer cell acquired mesenchymal, fibroblast-like morphology and shows reduced intercellular adhesion and increased motility [12]. Tumor progression is frequently associated with the down-regulation of E-cadherin, and up-regulation of vimentin, and several transcription factors including Snail, Twist and Slug $[12,25]$. A major clinical feature of NPC is frequent involvement of regional lymph nodes and distant organ metastasis. Even after treatment, increased invasiveness and migration of NPC during metastasis leads to poor outcomes and increased patient mortality [26]. Previous studies showed EMT molecular events associated with NPC metastasis were obvious in the absence of the Epstein-Barr virus genome [27].

The isolation of CSC from cancer cells was achieved successfully using several techniques. CSC can be enriched in spheres cultured in serum-free mediums supplemented with mitogens, such as the basic fibroblast growth factor (bFGF) and epidermal growth factor (EGF) $[13,28,29]$. Such cancer cells grown in a suspended condition exhibit resistance to anoikis, resulting in acquisition of ability to survive and proliferate [30].

We quantified and enriched CSC within NPC cell lines and subsequently characterized their phenotypical and functional properties, such as invasion capacity and epithelial-mesenchymal transition (EMT). These spheres contain putative CSC and can provide niches for the maintenance and growth of cancer cells. The EMT-type cells share many biological characteristics of CSC, which are linked closely with tumor recurrence and metastasis.

Previous studies exploited EGCG as a potential agent for prevention and treatment of cancers. EGCG suppresses the expression of HSP70 and HSP90, and exhibits anti-tumor activity in vitro and in vivo [31]. Moreover, in addition to EGCG-induced apoptosis effects, previous researches have recognized that EGCG-induced p21, p53, p16, and p27 expression, which is associated with negative regulations of cell cycle progression [32-34]. However, there were limited reports about EGCG regulation effects on CSC. Our study proposes phenotype changes 


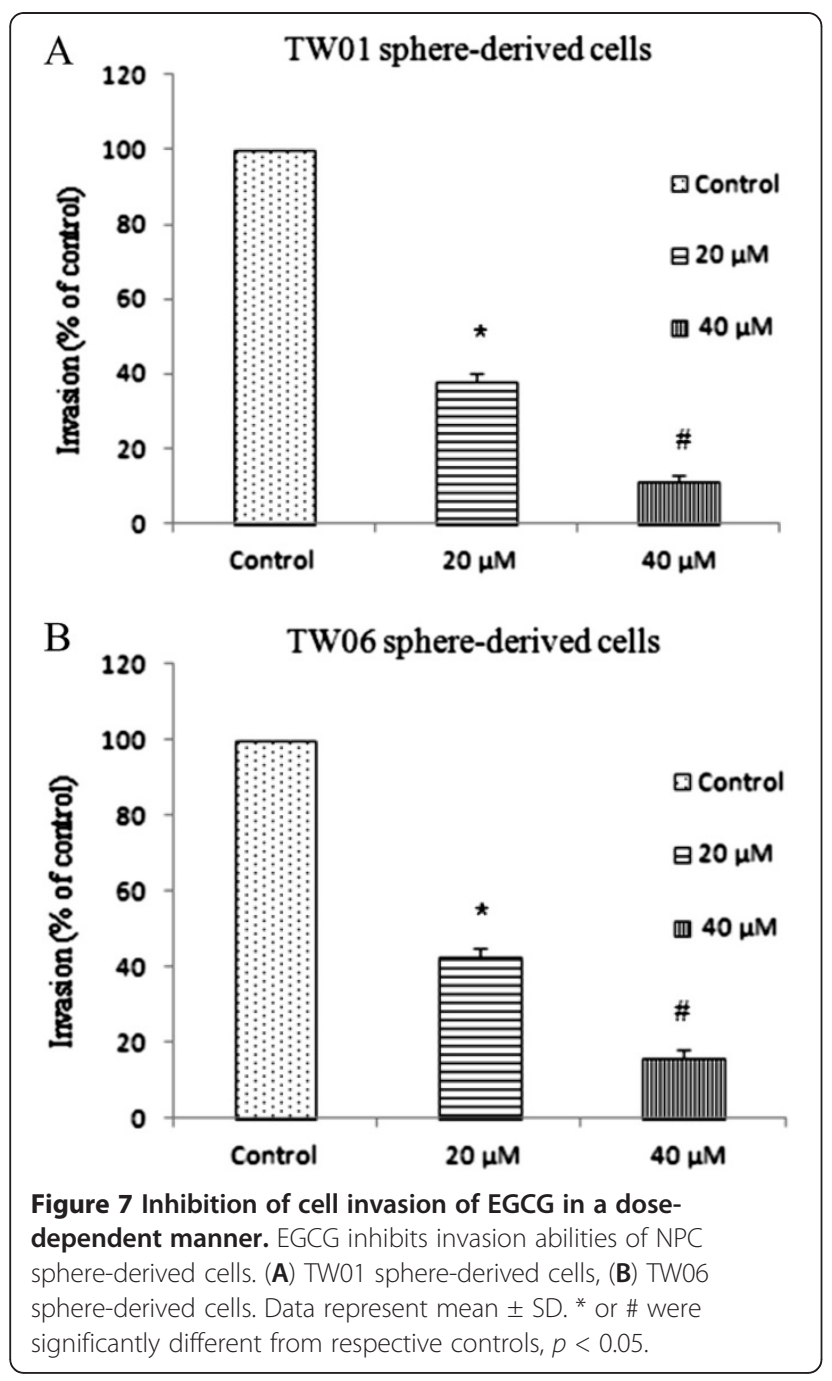

and functional consequences, by which the inhibitory effects of EGCG on CSC properties of NPC are seen.

Our designed experiments recognized the effects of EGCG on isolated sphere cells with anchorageindependent growth, and obtained novel findings. We discovered EGCG inhibits growth and induces apoptosis in parental NPC cells, but is inefficient in sphere-derived cells. Relative chemo-resistant NPC cells in spherederived cells, in accordance to Chen's study on nonadhesive culture systems exhibiting CSC characteristics, can be used in cancer research [35].

On the other hand, EGCG can inhibit sphere formation and regulate invasive-like phenotypes characterized by EMT of cells grown in serum free non-adherent cultures. In addition, it inhibited colony formation of sphere-derived cells grown in agar in a dose-dependent manner. This implicated that EGCG can be effective in suppressing the self-renewal capacity of NPC CSC. Furthermore, at the mRNA level, the expression of stemness maintaining transcription factors Klf- 4 and Oct- 4 were inhibited by EGCG. Several studies suggested Klf-4 and Oct-4 play important roles in maintaining stem cell identity, and are responsible for increased cell migration and invasion [36,37]. Our study further showed that EGCG inhibits the factors required for maintaining the self-renewal capacity in NPC CSC.

Because CSCs appear to have a significant role in invasion and metastasis, we measured the effects of EGCG on invasion and migration of NPC sphere-derived cells. The results showed that invasive and migratory behaviors were significantly reduced in the EGCG-treated group in a dose-dependent manner. This implied EGCG efficiently suppressed the metastasis ability in NPC CSC. Recent studies have also described the inhibitory activities of EGCG targeting cancer stem cells, which can suppress CSC self-renewal properties and block their migration and invasion $[38,39]$.

Enhanced EMT characteristics are associated with poor outcomes and declined survival in patients with NPC [40]. We examined EGCG regulating translational levels of EMT expression by TW01 spherederived cells. The western blot result showed EGCG inhibits the expression of vimentin, Snail, and is accompanied by the upregulation of E-cadherin. Immunofluorescence staining showed that vimentin expression in sphere-derived cells was decreased in
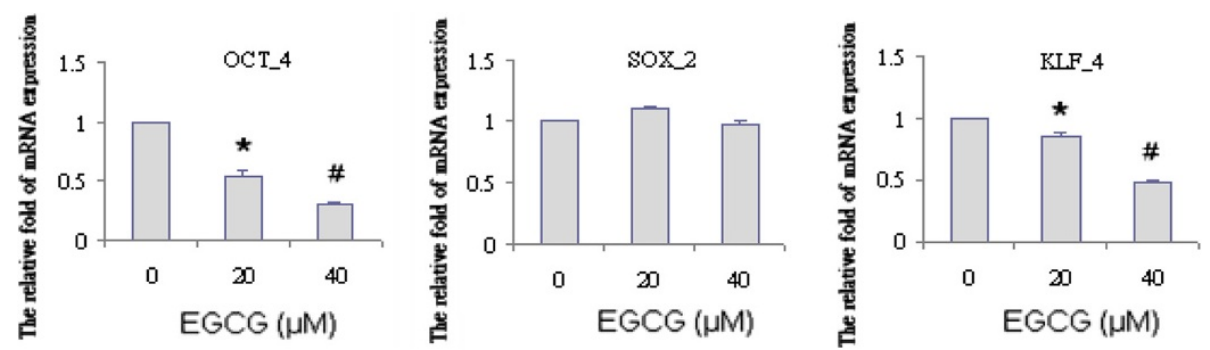

Figure 8 Expression of stemness genes was blocked by EGCG. The expression of stem cell genes (Oct4, Sox2, Klf4) of TW01 sphere-derived cells treated with EGCG was measured by the q-RT-PCR. Decreasing expression of OCT4 and KIf4 was accompanied with EGCG dosage. Data is represented mean $\pm \mathrm{SD} .{ }^{*}$ or \# means significantly different from respective controls, $p<0.05$. 

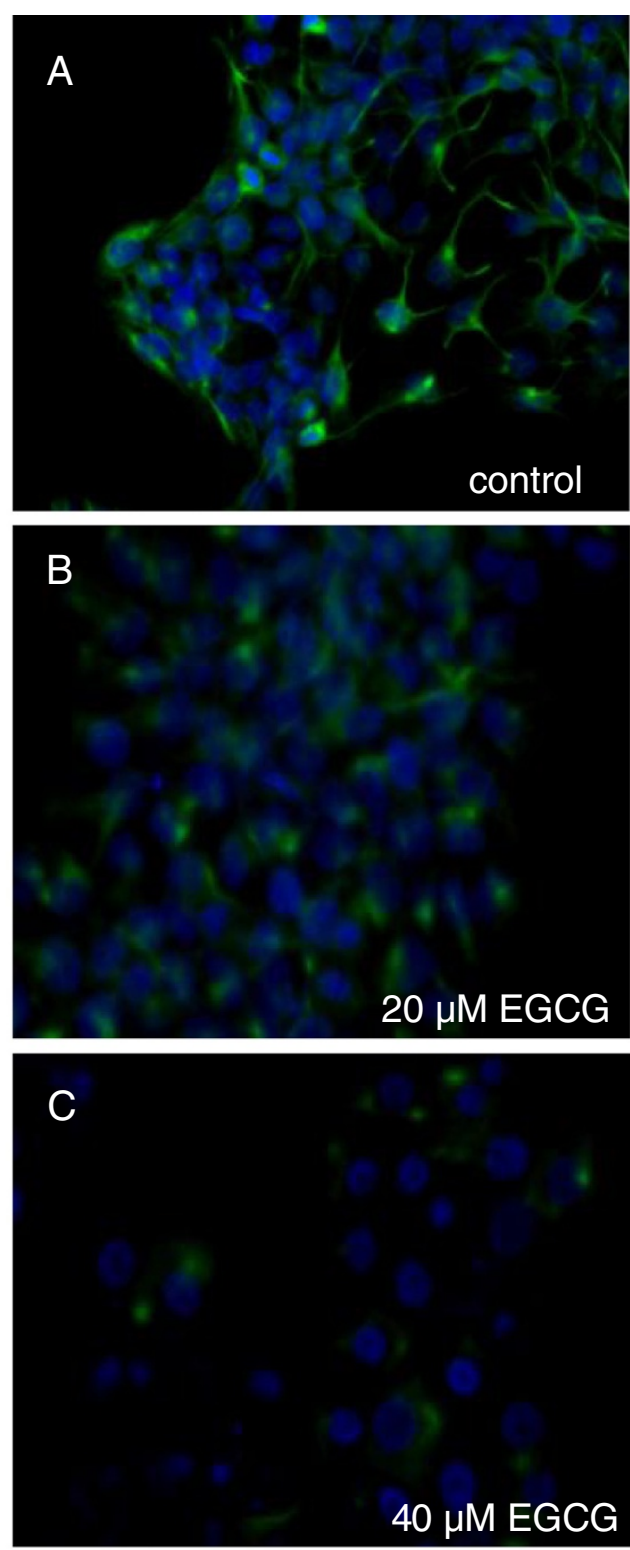

D

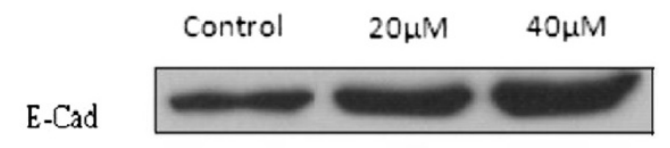

vimentin

Snail
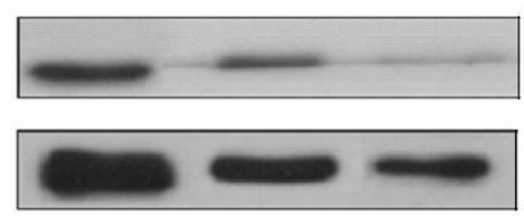

GAPDH
Figure 9 Effect of EGCG on EMT phenotype. Mesenchymal cell marker, vimentin expression was assayed by immunofluorescence staining in TW01 sphere-derived cells $(\mathbf{A} \sim \mathbf{C})$. (A) control group (B) treated with $20 \mu \mathrm{M}$ EGCG $(\mathbf{C})$ treated with $40 \mu \mathrm{M}$ EGCG. EGCG could decrease the expression of vimentin. Green fluorescent signal represents vimentin expression. Blue color is DAPI stain for nucleus. (D) Western blot signals in TW01 sphere-derived cells show decreased expression of vimentin and snail and increased expression of E-cadh (e-cadherin) after EGCG treatment.

the EGCG group compared to the control group. These results showed that EGCG may block NPC early metastasis signaling. Dysregulation of E-cadherin and $\beta$-catenin functions in cell-cell adhesion is common in NPC, and correlates with advanced stage disease and lymph node metastasis [41]. Our results further showed that EGCG inhibited NPC invasion and migration, correlating with the elevation of Ecadherin levels in NPC sphere-derived cells. E-cadherin is an important cell adhesion molecule, and has a key role in the early stage of tumor metastasis. Further investigation is required to study how EGCG modulates the expression of E-cadherin proteins.

Research regarding the use of EGCG against cancer has been proposed, however, most concentrations of EGCG used in cell lines are higher than the plasma concentrations observed in people after consuming tea because of its limited bioavailability in vivo [42]. To increase bioavailability and stability of EGCG, particular studies have elucidated structure-modified compounds with higher efficacy, which also possess anticancer activities $[39,43]$. Previous research has shown evidence of EGCG-induced apoptosis and cytotoxicity specifically on cancer cells, but not on normal cells under the same EGCG treatment $[44,45]$. This suggests that EGCG serves as an acceptable safety agent.

Overall, our findings further clarify the anti-cancer effects of EGCG, which can eliminate the stem-cell characteristics of NPC, and inhibit epithelial-mesenchymal transition (EMT), partially because of regulated EMTrelated proteins and lessen the migratory and invasive abilities of NPC. EGCG may be potentially effective in preventing the recurrence and metastasis of NPC in combination with standard treatment.

\section{Conclusions}

Our data shows that NPC cells with sphere formations possess the properties of CSC including EMT signatures, and their self-renewal capacities and invasive characteristics are inhibited by EGCG. This data supports the pivotal role of EGCG as a dietary compound targeting $\mathrm{NPC}$, and may decrease recurrence and metastasis in nasopharyngeal carcinoma cells. 


\section{Abbreviations}

EGCG: Epigallocathechin gallate; CSC: Cancer stem-like cell; TIC: Tumorinitiating cell; NPC: Nasopharyngeal carcinoma; EMT: Epithelial-mesenchymal transition; MTT: 3-(4,5-dimethylthiazol-2-yl)-2,5-diphenyltetrazolium bromide; MMP: Matrix metalloproteinases; BME: Basement membrane extract.

\section{Competing interests}

The authors have declared no conflict and financial competing of interests.

\section{Authors' contributions}

CHL: designed the study, performed experimental works, analyzed data, and generated the figures and manuscript. YAS: designed the protocol for NPC CSC selection and sphere formation. PHH: data analysis and performed the statistical analysis. YBY: intellectual support and coordination of the study. YJC: Project design, project coordination and manuscript preparation. All authors have read and approved of the final version of the manuscript.

\section{Acknowledgements}

This project was supported by grants from the National Science Council, Taiwan (NSC-99-2314-B-010-006-MY3, NSC-96-2320-B-010-035-MY2), Department of Health, Taipei City (Grant No. 10001-62-030), and National Yang Ming University, Ministry of Education, Aim for the Top University Plan.

\section{Author details}

${ }^{1}$ Institute of Clinical Medicine, National Yang-Ming University, Taipei, Taiwan. ${ }^{2}$ Department of Pediatrics, Zhongxing Branch, Taipei City Hospital, Taipei, Taiwan. ${ }^{3}$ Institute of Biochemistry and Molecular Biology, National Yang-Ming University, Taipei, Taiwan. ${ }^{4}$ Department of Internal Medicine, Ditmanson Medical Foundation Chia-yi Christian Hospital, Chia-yi, Taiwan. ${ }^{5}$ Division of Hematology and Oncology, Department of Medicine, Taipei Veterans General Hospital, Taipei, Taiwan. ${ }^{6}$ Department of Pediatrics, Renai Branch, Taipei City Hospital, Taipei, Taiwan. ${ }^{7}$ Department of Life Sciences and Institute of Genome Sciences, National Yang-Ming University, Taipei, Taiwan.

\section{Received: 8 May 2012 Accepted: 22 October 2012}

Published: 30 October 2012

\section{References}

1. Cheung F, Chan O, Ng WT, Chan L, Lee A, Pang SW: The prognostic value of histological typing in nasopharyngeal carcinoma. Oral Oncol 2012, 48(5):429-433.

2. Lo KW, To KF, Huang DP: Focus on nasopharyngeal carcinoma. Cancer Cell 2004, 5(5):423-428.

3. Yeh SA, Tang Y, Lui CC, Huang YJ, Huang EY: Treatment outcomes and late complications of 849 patients with nasopharyngeal carcinoma treated with radiotherapy alone. Int J Radiat Oncol Biol Phys 2005, 62(3):672-679.

4. Jordan CT, Guzman ML, Noble M: Cancer stem cells. N Engl J Med 2006, 355(12):1253-1261

5. Dalerba P, Cho RW, Clarke MF: Cancer stem cells: models and concepts. Annu Rev Med 2007, 58:267-284.

6. Frame FM, Maitland NJ: Cancer stem cells, models of study and implications of therapy resistance mechanisms. Adv Exp Med Biol 2011, 720:105-118.

7. Wang J, Guo LP, Chen LZ, Zeng YX, Lu SH: Identification of cancer stem cell-like side population cells in human nasopharyngeal carcinoma cell line. Cancer Res 2007, 67(8):3716-3724.

8. Su J, Xu XH, Huang Q, Lu MQ, Li DJ, Xue F, Yi F, Ren JH, Wu YP: Identification of cancer stem-like CD44+ cells in human nasopharyngeal carcinoma cell line. Arch Med Res 2011, 42(1):15-21.

9. Mani SA, Guo W, Liao MJ, Eaton EN, Ayyanan A, Zhou AY, Brooks M, Reinhard F, Zhang CC, Shipitsin M, et al: The epithelial-mesenchymal transition generates cells with properties of stem cells. Cell 2008, 133(4):704-715.

10. Kong D, Banerjee S, Ahmad A, Li Y, Wang Z, Sethi S, Sarkar FH: Epithelial to mesenchymal transition is mechanistically linked with stem cell signatures in prostate cancer cells. PLoS One 2010, 5(8):e12445.

11. Hollier BG, Evans K, Mani SA: The epithelial-to-mesenchymal transition and cancer stem cells: a coalition against cancer therapies. J Mammary Gland Biol Neoplasia 2009, 14(1):29-43.
12. Polyak K, Weinberg RA: Transitions between epithelial and mesenchymal states: acquisition of malignant and stem cell traits. Nat Rev Cancer 2009, 9(4):265-273.

13. Lee J, Kotliarova S, Kotliarov Y, Li A, Su Q, Donin NM, Pastorino S, Purow BW, Christopher N, Zhang W, et al: Tumor stem cells derived from glioblastomas cultured in bFGF and EGF more closely mirror the phenotype and genotype of primary tumors than do serum-cultured cell lines. Cancer Cell 2006, 9(5):391-403.

14. Gupta S, Hastak K, Afaq F, Ahmad N, Mukhtar H: Essential role of caspases in epigallocatechin-3-gallate-mediated inhibition of nuclear factor kappa B and induction of apoptosis. Oncogene 2004, 23(14):2507-2522.

15. Ahmad N, Gupta S, Mukhtar H: Green tea polyphenol epigallocatechin-3gallate differentially modulates nuclear factor kappaB in cancer cells versus normal cells. Arch Biochem Biophys 2000, 376(2):338-346.

16. Shankar S, Ganapathy S, Srivastava RK: Green tea polyphenols: biology and therapeutic implications in cancer. Front Biosci 2007, 12:4881-4899.

17. Shankar S, Suthakar G, Srivastava RK: Epigallocatechin-3-gallate inhibits cell cycle and induces apoptosis in pancreatic cancer. Front Biosci 2007, 12:5039-5051.

18. Adhami VM, Ahmad N, Mukhtar H: Molecular targets for green tea in prostate cancer prevention. J Nutr 2003, 133(7 Suppl):2417S-2424S.

19. Wang $X$, Hao MW, Dong K, Lin F, Ren JH, Zhang HZ: Apoptosis induction effects of EGCG in laryngeal squamous cell carcinoma cells through telomerase repression. Arch Pharm Res 2009, 32(9):1263-1269.

20. Chan MM, Soprano KJ, Weinstein K, Fong D: Epigallocatechin-3-gallate delivers hydrogen peroxide to induce death of ovarian cancer cells and enhances their cisplatin susceptibility. J Cell Physiol 2006, 207(2):389-396.

21. Thangapazham RL, Singh AK, Sharma A, Warren J, Gaddipati JP, Maheshwari RK: Green tea polyphenols and its constituent epigallocatechin gallate inhibits proliferation of human breast cancer cells in vitro and in vivo. Cancer Lett 2007, 245(1-2):232-241.

22. Fong $D$, Yeh $A$, Naftalovich $R$, Choi TH, Chan MM: Curcumin inhibits the side population (SP) phenotype of the rat C6 glioma cell line: towards targeting of cancer stem cells with phytochemicals. Cancer Lett 2010, 293(1):65-72.

23. Burnett J, Newman B, Sun D: Targeting cancer stem cells with natural products. Curr Drug Targets 2012, 13(8):1054-1064.

24. Hu FW, Tsai LL, Yu CH, Chen PN, Chou MY, Yu CC: Impairment of tumor-initiating stem-like property and reversal of epithelial-mesenchymal transdifferentiation in head and neck cancer by resveratrol treatment. Mol Nutr Food Res 2012, 56(8):1247-1258.

25. Thiery JP, Acloque H, Huang RY, Nieto MA: Epithelial-mesenchymal transitions in development and disease. Cell 2009, 139(5):871-890.

26. Bensouda Y, Kaikani W, Ahbeddou N, Rahhali R, Jabri M, Mrabti H, Boussen $\mathrm{H}$, Errihani $\mathrm{H}$ : Treatment for metastatic nasopharyngeal carcinoma. Eur Ann Otorhinolaryngol Head Neck Dis 2011, 128(2):79-85.

27. Lin J-C, Liao S-K, Lee E-H, Hung M-S, Sayion Y, Chen H-C, Kang C-C, Huang L-S, Cherng J-M: Molecular events associated with epithelial to mesenchymal transition of nasopharyngeal carcinoma cells in the absence of Epstein-Barr virus genome. J Biomed Sci 2009, 16(1):105.

28. Hueng DY, Sytwu HK, Huang SM, Chang C, Ma HI: Isolation and characterization of tumor stem-like cells from human meningiomas. J Neurooncol 2011, 104(1):45-53.

29. Zhong Y, Guan K, Guo S, Zhou C, Wang D, Ma W, Zhang Y, Li C, Zhang S: Spheres derived from the human SK-RC-42 renal cell carcinoma cell line are enriched in cancer stem cells. Cancer Lett 2010, 299(2):150-160.

30. Liotta LA, Kohn E: Anoikis: cancer and the homeless cell. Nature 2004 , 430(7003):973-974.

31. Tran PL, Kim SA, Choi HS, Yoon JH, Ahn SG: Epigallocatechin-3-gallate suppresses the expression of HSP70 and HSP90 and exhibits anti-tumor activity in vitro and in vivo. BMC Cancer 2010, 10:276.

32. Liang YC, Lin-Shiau SY, Chen CF, Lin JK: Inhibition of cyclin-dependent kinases 2 and 4 activities as well as induction of Cdk inhibitors p21 and p27 during growth arrest of human breast carcinoma cells by (-)-epigallocatechin-3gallate. J Cell Biochem 1999, 75(1):1-12.

33. Gupta S, Ahmad N, Nieminen AL, Mukhtar H: Growth inhibition, cell-cycle dysregulation, and induction of apoptosis by green tea constituent (-)-epigallocatechin-3-gallate in androgen-sensitive and androgen-insensitive human prostate carcinoma cells. Toxicol Appl Pharmacol 2000, 164(1):82-90.

34. Gupta S, Hussain T, Mukhtar H: Molecular pathway for (-)-epigallocatechin-3-gallate-induced cell cycle arrest and apoptosis 
of human prostate carcinoma cells. Arch Biochem Biophys 2003, 410(1):177-185.

35. Chen SF, Chang YC, Nieh S, Liu CL, Yang CY, Lin YS: Nonadhesive culture system as a model of rapid sphere formation with cancer stem cell properties. PLoS One 2012, 7(2):e31864.

36. Yu F, Li J, Chen H, Fu J, Ray S, Huang S, Zheng H, Ai W: Kruppel-like factor 4 (KLF4) is required for maintenance of breast cancer stem cells and for cell migration and invasion. Oncogene 2011, 30(18):2161-2172.

37. Kim RJ, Nam JS: OCT4 Expression Enhances Features of Cancer Stem Cells in a Mouse Model of Breast Cancer. Lab Anim Res 2011, 27(2):147-152.

38. Tang S-N, Singh C, Nall D, Meeker D, Shankar S, Srivastava RK: The dietary bioflavonoid quercetin synergizes with epigallocathechin gallate (EGCG) to inhibit prostate cancer stem cell characteristics, invasion, migration and epithelial-mesenchymal transition. J Mol Signaling 2010, 5(1):14.

39. Chen D, Pamu S, Cui Q, Chan TH, Dou QP: Novel epigallocatechin gallate (EGCG) analogs activate AMP-activated protein kinase pathway and target cancer stem cells. Bioorg Med Chem 2012, 20(9):3031-3037.

40. Luo W, Fang W, Li S, Yao K: Aberrant expression of nuclear vimentin and related epithelial-mesenchymal transition markers in nasopharyngeal carcinoma. Int J Canc 2012, 131(8):1863-1873.

41. Yip WK, Seow HF: Activation of phosphatidylinositol 3-kinase/Akt signaling by EGF downregulates membranous E-cadherin and beta-catenin and enhances invasion in nasopharyngeal carcinoma cells. Cancer Lett 2012, 318(2):162-172.

42. Yang CS, Sang S, Lambert JD, Lee MJ: Bioavailability issues in studying the health effects of plant polyphenolic compounds. Mol Nutr Food Res 2008, 52(Suppl 1):S139-151.

43. Osanai K, Landis-Piwowar KR, Dou QP, Chan TH: A para-amino substituent on the D-ring of green tea polyphenol epigallocatechin-3-gallate as a novel proteasome inhibitor and cancer cell apoptosis inducer. Bioorg Med Chem 2007, 15(15):5076-5082.

44. Chen ZP, Schell JB, Ho CT, Chen KY: Green tea epigallocatechin gallate shows a pronounced growth inhibitory effect on cancerous cells but not on their normal counterparts. Cancer Lett 1998, 129(2):173-179.

45. Yamamoto T, Hsu S, Lewis J, Wataha J, Dickinson D, Singh B, Bollag WB, Lockwood P, Ueta E, Osaki T, et al: Green tea polyphenol causes differential oxidative environments in tumor versus normal epithelial cells. J Pharmacol Exp Ther 2003, 307(1):230-236.

doi:10.1186/1472-6882-12-201

Cite this article as: Lin et al.: Epigallocathechin gallate, polyphenol present in green tea, inhibits stem-like characteristics and epithelialmesenchymal transition in nasopharyngeal cancer cell lines. BMC Complementary and Alternative Medicine 2012 12:201.

\section{Submit your next manuscript to BioMed Central and take full advantage of:}

- Convenient online submission

- Thorough peer review

- No space constraints or color figure charges

- Immediate publication on acceptance

- Inclusion in PubMed, CAS, Scopus and Google Scholar

- Research which is freely available for redistribution

Submit your manuscript at www.biomedcentral.com/submit
C Biomed Central 